\title{
Model-based Sensor Fault Detection in an Autonomous Solar-powered Aircraft
}

\author{
Paulo Victor Padrao Lopes , Liu Hsu , Michael Vilzmann*, and Konstantin Kondak* \\ Departament of Electrical Engineering, COPPE, Federal University of Rio de Janeiro, Brazil \\ E-mail: paulo.padrao@iff.edu.br \\ *Department of Robotics and Mechatronics, German Aerospace Center, Germany \\ *E-mail: michael.vilzmann@dlr.de
}

\begin{abstract}
Developed by Elektra Solar, an official spin-off of the DLR Institute for Robotics and Mechatronics (DLR-RMC), the Elektra 2 is a solar-powered autonomous aircraft designed to endure long distances as well as high altitudes. The main motivation of this work is to develop a sensor fault detection and reconfiguration (FDR) approach to be applied to the Elektra 2 Aircraft. Currently, the Elektra 2 Solar aircraft provides a simple limit-checking of certain IMU measurements such as aircraft angular velocities and pitch, roll and yaw angles. In the proposed FDR approach, residual generation for both longitudinal and lateral dynamics of the aircraft is achieved based on the design of Kalman filters. Combined with the sequential probability ratio test (SPRT) as a decision function, selected aircraft sensor residuals are evaluated using an adaptive threshold with low sensitivity to control signal variation and noise and high sensitivity to fault symptoms. Due to the critical aspects of IMU faults, the reconfiguration action is defined as switching from primary IMU to backup IMU in case of fault occurrence.
\end{abstract}

Keywords: Sensor Fault Detection, Fault Reconfiguration, Autonomous Aircraft Systems

\section{Introduction}

Sensors are the most critical components for aircraft safety and it is well known that hardware redundancy plays a fundamental role in fault tolerant systems [1], [2]. In order to reduce cost limitations and system complexity, analytical redundancy approach arises as a suitable option for navigation sensor fault detection of such systems [3], [4]. Analytical redundancy uses a mathematical model of the monitored system and it is here achieved by properly designed estimators that provide robust residual generation for fault detection framework [5]. These residuals are then evaluated using an adaptive threshold with low sensitivity to control signal variation and noise and high sensitivity to fault symptoms. The main motivation of this work is to develop a fault detection and reconfiguration approach to be applied to the Elektra 2 Aircraft (fig. 11. Currently, the aircraft provides a simple limit-checking of certain measurements such as aircraft angular velocities and pitch, roll and yaw angles. Developed by Elektra Solar, an official spin-off of the Institute for Robotics and Mechatronics at the German Aerospace Center, the Elektra 2 is a solar-powered autonomous aircraft designed to endure long distances as well as high altitudes.

\section{Proposed Fault Detection Approach}

The proposed fault detection and reconfiguration approach for the Elektra 2 aircraft is illustrated in figure 2 Residual gen-

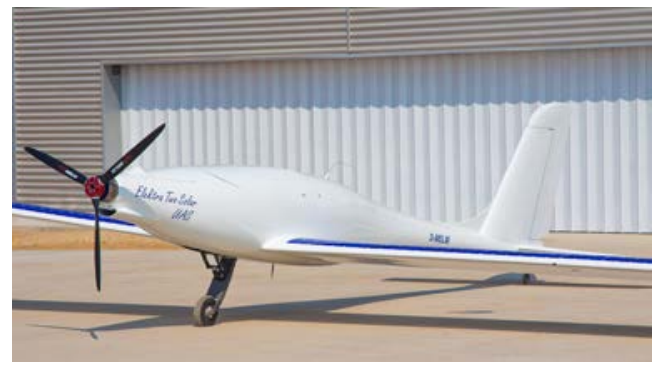

Figure 1: Autonomous solar-powered aircraft: Elektra 2.

eration for both longitudinal and lateral dynamics of the aircraft is achieved based on the design of Kalman filters [6]. The sequential probability ratio test (SPRT) is then used as a decision function to be evaluated with adaptive thresholds (ATLMS) for each of the aircraft measurements. Due to the critical aspects of IMU faults, the reconfiguration action is defined as switching from primary IMU to backup IMU in case of fault occurrence. Therefore, a hot standby reconfiguration scheme is used for sensor fault tolerant purposes.

\subsection{Aircraft Model and Flight Conditions}

The six-degree-of-freedom, linearized model of the aircraft is decoupled into longitudinal and lateral-directional dynamics as follows 


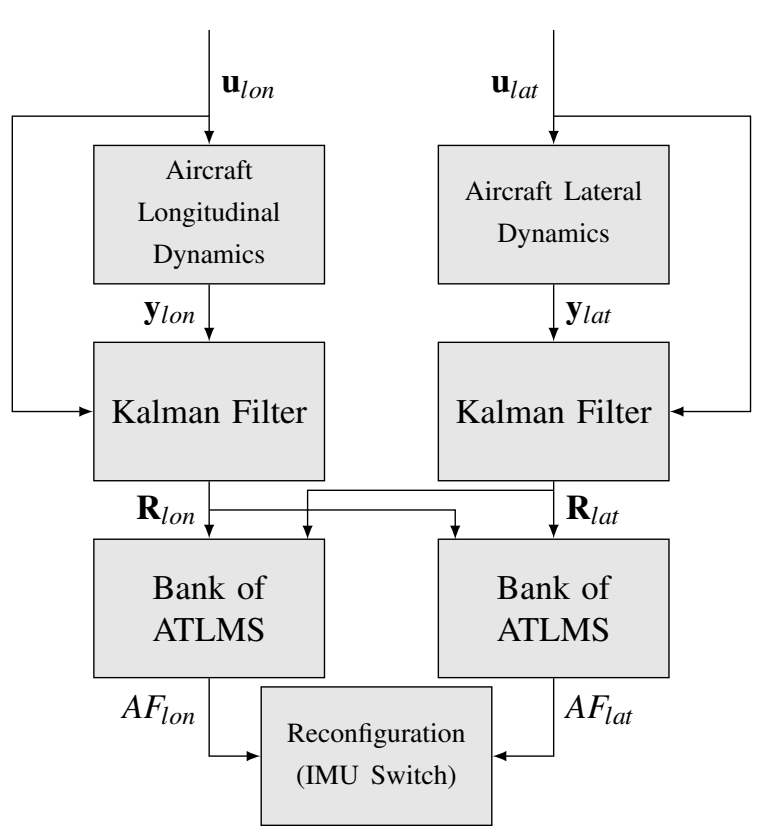

Figure 2: $\mathbf{u}_{l o n}$ and $\mathbf{u}_{l a t}$ are the control signals for the decoupled longitudinal and lateral aircraft dynamics, respectively. $\mathbf{y}_{\text {lon }}$ and $\mathbf{y}_{\text {lat }}$ are the aircraft measurements from longitudinal and lateral aircraft dynamics, respectively. $\mathbf{R}_{\text {lon }}$ and $\mathbf{R}_{\text {lat }}$ are the aircraft residuals from longitudinal and lateral aircraft dynamics, respectively. $A F_{l o n}$ and $A F_{l a t}$ refer to the longitudinal and lateral alarm flags, respectively.

$$
\begin{gathered}
{\left[\begin{array}{c}
\dot{\mathbf{x}}_{\text {lon }} \\
\dot{\mathbf{x}}_{\text {lat }}
\end{array}\right]=\left[\begin{array}{cc}
\mathbf{A}_{\text {lon }} & 0 \\
0 & \mathbf{A}_{\text {lat }}
\end{array}\right]\left[\begin{array}{c}
\mathbf{x}_{\text {lon }} \\
\mathbf{x}_{\text {lat }}
\end{array}\right]+\left[\begin{array}{cc}
\mathbf{B}_{\text {lon }} & 0 \\
0 & \mathbf{B}_{\text {lat }}
\end{array}\right]\left[\begin{array}{l}
\mathbf{u}_{\text {lon }} \\
\mathbf{u}_{\text {lat }}
\end{array}\right]} \\
{\left[\begin{array}{c}
\mathbf{y}_{\text {lon }} \\
\mathbf{y}_{\text {lat }}
\end{array}\right]=\left[\begin{array}{cc}
\mathbf{C}_{\text {lon }} & 0 \\
0 & \mathbf{C}_{\text {lat }}
\end{array}\right]\left[\begin{array}{l}
\mathbf{x}_{\text {lon }} \\
\mathbf{x}_{\text {lat }}
\end{array}\right]}
\end{gathered}
$$

The longitudinal dynamics describes the aircraft motion in the $(x, z)$ body plane and it has 3 degrees of freedom: pitch, longitudinal motion and vertical motion.

The state vector for longitudinal motion is given by

$$
\mathbf{x}_{\text {lon }} \triangleq(u, w, q, \theta, h)^{T}
$$

where $u$ and $w$ are the linear velocities along the $x$ and $z$ axes respectively,in body frame; $q$ corresponds to the pitch rate; $\theta$ indicates the pitch angle; $h$ indicates the altitude above ground the aircraft. The respective input vector is defined as

$$
\mathbf{u}_{l o n} \triangleq\left(\delta_{e}, \delta_{t}\right)^{T} .
$$

where $\delta_{e}$ and $\delta_{t}$ are the elevator and throttle commands, respectively.

The lateral dynamics describes the aircraft motion around the $z$-axis and consists of roll, yaw and lateral motion. The state vector for lateral motion is given by

$$
\mathbf{x}_{l a t} \triangleq(v, p, r, \phi, \psi)^{T}
$$

where $v$ is the linear velocities along the $y$ axis, in body frame; $p$ and $r$ correspond to the roll and yaw rates, respectively; $\phi$ and $\psi$ the roll angle and yaw angle, respectively;

and the respective input vector is defined as

$$
\mathbf{u}_{l a t} \triangleq\left(\delta_{a}, \delta_{r}\right)^{T}
$$

where $\delta_{a}$ and $\delta_{r}$ are the aileron and rudder commands, respectively. All states and measurements are provided in the International System of Units (SI). The measured output vectors used here are rewritten in terms of $V_{a}, \alpha$, and $\beta$ as follows

$$
\begin{gathered}
\mathbf{y}_{\text {lon }_{m}} \triangleq\left(V_{a}, \alpha, q, \theta, h\right)^{T} \\
\mathbf{y}_{\text {lat }_{m}} \triangleq(\beta, p, r, \phi, \psi)^{T} .
\end{gathered}
$$

where $V_{a}$ is the true airspeed, $\alpha$ is the angle of attack and $\beta$ the sideslip angle, which are measurable by air data sensors on the aircraft as in equation (9)

$$
\begin{aligned}
V_{a} & =\sqrt{u^{2}+v^{2}+w^{2}} \\
\alpha & =\arctan \left(\frac{w}{u}\right) \\
\beta & =\arcsin \left(\frac{v}{V_{a}}\right)
\end{aligned}
$$

\subsection{Proposed IMU Faults}

In this work, three different types of additive IMU faults are presented in table 1 .

- Abrupt Fault: modelled as a step-wise function. It represents bias in the monitored signal;

- Incipient Fault: modelled by ramp signals. It represents drift of the monitored signal;

- Extra Noise Fault: modelled by an abrupt change of the signal standard deviation.

Table 1: Proposed IMU Faults

\begin{tabular}{c|c|c}
\hline Fault & Measurement & Amplitude \\
\hline Incipient & $\theta$ & $0.2 \mathrm{rad} / \mathrm{s}$ \\
Extra Noise & $q$ & $\mathscr{N}(\bar{\mu}=0, \sigma=0.01)$ \\
Abrupt & $\phi$ & $0.2 \mathrm{rad}$ \\
\hline
\end{tabular}

\subsection{Adaptive Threshold based on the LMS Filter (ATLMS)}

The adaptive threshold (ATLMS) presented here is based on the technique exposed in [7]. The ATLMS technique allows the threshold to be tuned by changing well known parameters independently of the application and is based on SPRT algorithm, the LMS (Least-Mean-Square) algorithm, and residual sensitivity. The residual is said to be fault-sensitive if 
it is highly influenced by fault occurrence and consequently deviates significantly from non-faulty mode. In contrast, a residual is said to be fault-insensitive if it is not influenced by fault occurrence or such influence is negligible in comparison with the sensitive residual. Differently from the application proposed by [7] and taking advantage of the decoupling nature of the system, the design of sensitive and insensitive residuals was obtained by the decoupling of the lateral and longitudinal aircraft linear models. Once this is not always possible for other systems, sensitive and insensitive residuals can also be accomplished by structured residual technique as presented in [8] or by a bank of observers/estimators as in [9], for example.

The basic idea behind the proposed adaptive threshold is to use the adaptability of the LMS algorithm to reinitialize the SPRT algorithm automatically (fig. 3) and the requirements to the dynamic behavior of such threshold are (i) low sensitivity to control signal variation; (ii) low sensitivity to noise and (iii) high sensitivity to faulty residuals.

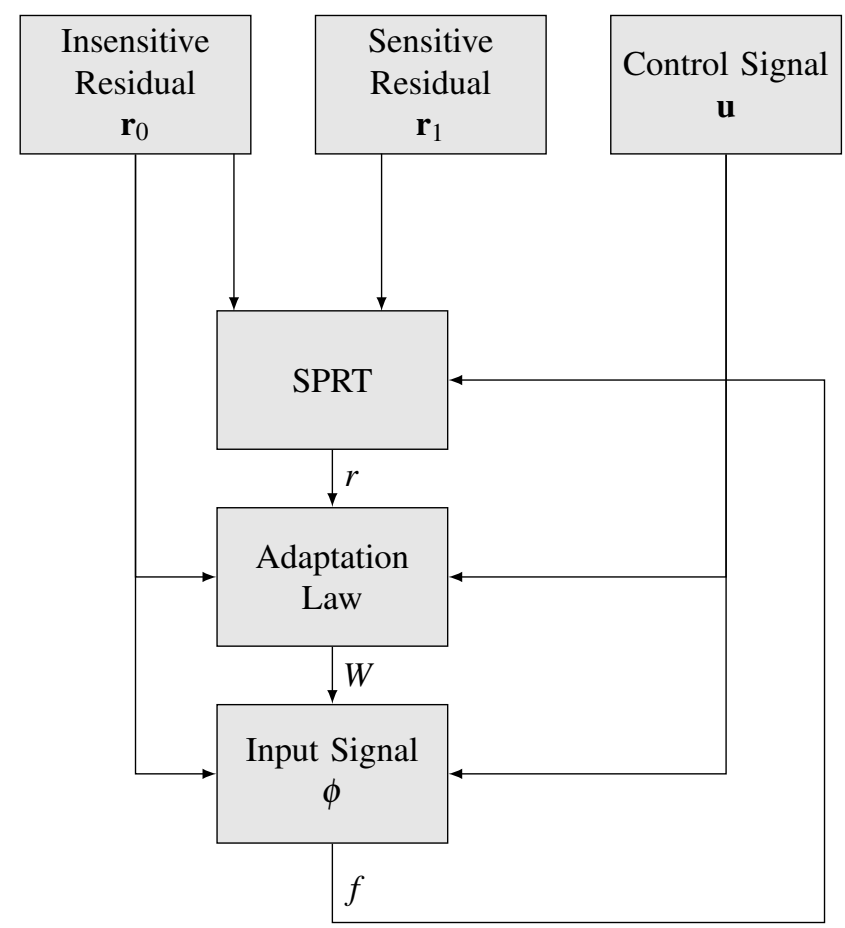

Figure 3: ATLMS Scheme: $\mathbf{u}$ is the control signal vector, $r_{0}$ is the insensitive residual, $r_{1}$ is the sensitive residual, $r$ is the instantaneous SPRT response, $\mathrm{W}$ is the weight vector of the $L M S$ algorithm, and $\phi$ is the input signal vector composed of $\mathbf{u}$ and recursions of the insensitive residual. A fault alarm flag is raised if the adaptive threshold $f$ crosses the insensitive residual $r_{0}$.

The adaptive threshold $f$ is computed at each sample period as:

$$
f_{k}=\phi_{k}^{T} \mathbf{W}_{k}
$$

where $\mathbf{W}$ is the weight vector of the LMS algorithm, and $\phi$ is the input signal vector composed of the control signal $\mathbf{u}$ and recursions of the insensitive residual $r_{0}$. The SPRT can be calculated as

$$
L_{k}=L_{k-1}+\ln \frac{p_{0}\left(r_{0 k} \mid r_{0 k-1}, \ldots, r_{0 k-d}\right)}{p_{1}\left(r_{1 k} \mid r_{1 k-1}, \ldots, r_{1 k-d}\right)}
$$

where $p_{0}$ and $p_{1}$ are the probability density function of the insensitive and sensitive residuals $r_{0}$ and $r_{1}$, respectively. Considering that $r_{0}$ and $r_{1}$ present normal distribution with zero mean and same variance $\sigma_{0}^{2}$, the ratio $\ln \left(\frac{p_{0}}{p_{1}}\right)$ can be simplified by $\frac{r_{0 k}^{2}-r_{1 k}^{2}}{2 \sigma_{0}^{2}}$. For each data sample, three decisions can be made:

- In favor of a faulty mode (Hypothesis $\mathscr{H}_{1}$ );

- In favor of a normal mode (Hypothesis $\mathscr{H}_{0}$ );

- There is insufficient information to decide in favor of faulty or normal mode.

For this reason, under different hypotheses, the expected value of the SPRT response presents trends in opposite directions as follows

$$
\begin{aligned}
& E\left\{\frac{r_{0 k}^{2}-r_{1 k}^{2}}{2 \sigma_{0}^{2}} \mid \mathscr{H}_{0}\right\}<0 \rightarrow \dot{L}_{k}<0 \\
& E\left\{\frac{r_{0 k}^{2}-r_{1 k}^{2}}{2 \sigma_{0}^{2}} \mid \mathscr{H}_{1}\right\}>0 \rightarrow \dot{L}_{k}>0
\end{aligned}
$$

where $\dot{L}_{k}=\frac{d E\left\{L_{k}\right\}}{d t}$.

The SPRT decision update is based on the difference between the adaptive threshold $f$ and the insensitive residual $r_{0}$ as follows

$$
\gamma=f-r_{0}
$$

The update term $\gamma$ should also be affected by a varying term $e$ as

$$
e=e_{0}-a \dot{L}_{k}
$$

where $\dot{L}_{k}$ is the expected trend of the SPRT, $e_{0}$ is the safe distance between the adaptive threshold $f$ and insensitive residual $r_{0}$, and $a$ is the sensitivity due to changes in the trend of the SPRT at instant $k$. More details about these tuning parameters are shown in section 2.4. Without loss of generality and for the sake of implementation procedures, the derivative of the expected value of $L_{k}, \dot{L}_{k}$, is simplified by the approximation $\dot{\tilde{L}}_{k}=\frac{L_{k}-L_{k-1}}{T_{s}}$, where the additional parameter $T_{s}$ is the sampling period. Thus, the objective function to the synthesis of the adaptive threshold is defined as

$$
\min \left(\gamma_{k}-e_{k}\right)^{2}
$$


and the LMS algorithm is used to find the optimal weight vector $\mathbf{W}_{k}^{*}$ that minimizes such objective function.

The quadratic performance index can be rewritten as

$$
\begin{aligned}
\left(\gamma_{k}-e_{k}\right)^{2} & =(\phi_{k}^{T} \mathbf{W}_{k}-\underbrace{r_{0 k}-e_{0}+\left(\frac{a}{2 T_{s} \sigma_{0}^{2}}\right)\left(r_{0 k}^{2}-r_{1 k}^{2}\right)}_{s_{k}})^{2} \\
& =\mathbf{W}_{k}^{T} \phi_{k} \phi_{k}^{T} \mathbf{W}_{k}-2 \phi_{k}^{T} \mathbf{W}_{k} s_{k}+s_{k}^{2}
\end{aligned}
$$

The weight vector $\mathbf{W}_{k}$ that minimizes the quadratic performance index can be found by the gradient method as in the LMS algorithm as follows

$$
\mathbf{W}_{k}=\mathbf{W}_{k-1}+\mu\left[-\frac{\partial\left(\gamma_{k}-e_{k}\right)^{2}}{\partial \mathbf{W}}\right]_{\mathbf{W}=\mathbf{W}_{k-1}}
$$

An approximate estimate of the gradient at each sampling instant is given by

$$
\frac{\partial\left(\gamma_{k}-e_{k}\right)^{2}}{\partial \mathbf{W}}=2\left(\gamma_{k}-e_{k}\right) \frac{\partial\left(\gamma_{k}-e_{k}\right)}{\partial \mathbf{W}}=2\left(\gamma_{k}-e_{k}\right) \phi_{k}
$$

and its proof is provided below.

Proof. The expected value of equation 16 is

$E\left\{\left(\gamma_{k}-e_{k}\right)^{2}\right\}=E\left\{\mathbf{W}_{k}^{T} \phi_{k} \phi_{k}^{T} \mathbf{W}_{k}\right\}-E\left\{2 \phi_{k}^{T} \mathbf{W}_{k} s_{k}\right\}+E\left\{s_{k}^{2}\right\}$

and its partial derivative regarding the weight vector $\mathbf{W}$ is

$$
\begin{aligned}
\frac{\partial E\left\{\left(\gamma_{k}-e_{k}\right)^{2}\right\}}{\partial \mathbf{W}} & =2 E\left\{\phi_{k} \mathbf{W}_{k} \phi_{k}^{T}\right\}-2 \underbrace{E\left\{\phi_{k}^{T} s_{k}\right\}}_{\Theta} \\
& =2\left[E\left\{\phi_{k} \mathbf{W}_{k} \phi_{k}^{T}\right\}-\Theta\right]
\end{aligned}
$$

The result presented in eq. 20 must be the same as the expected value of eq. (18), as follows

$$
\begin{aligned}
E\left\{2\left(\gamma_{k}-e_{k}\right) \phi_{k}\right\} & =2\left[E\left\{\gamma_{k} \phi_{k}\right\}-E\left\{e_{k} \phi_{k}\right\}\right] \\
& =2\left[E\left\{f_{k} \phi_{k}-r_{0 k} \phi_{k}\right\}-E\left\{\left(e_{0}-a \dot{\tilde{L}}_{k}\right) \phi_{k}\right\}\right] \\
& =2\left[E\left\{\phi_{k}^{T} \mathbf{W}_{k} \phi_{k}-r_{0 k} \phi_{k}\right\}-E\left\{\left(e_{0}-a \dot{\tilde{L}}_{k}\right) \phi_{k}\right\}\right] \\
& =2\left[E\left\{\phi_{k}^{T} \mathbf{W}_{k} \phi_{k}\right\}-E\left\{(\underbrace{r_{0 k}+e_{0}-a \dot{\tilde{L}}_{k}}_{s_{k}}) \phi_{k}\right\}\right] \\
& =2[E\left\{\phi_{k}^{T} \mathbf{W}_{k} \phi_{k}\right\}-\underbrace{E\left\{\phi_{k} s_{k}\right\}}_{\Theta}] \\
& =2\left[E\left\{\phi_{k}^{T} \mathbf{W}_{k} \phi_{k}\right\}-\Theta\right]
\end{aligned}
$$

This proves that

$$
\frac{\partial E\left\{\left(\gamma_{k}-e_{k}\right)^{2}\right\}}{\partial \mathbf{W}}=E\left\{2\left(\gamma_{k}-e_{k}\right) \phi_{k}\right\}=2\left[E\left\{\phi_{k} \phi_{k}^{T}\right\} \mathbf{W}_{k}-\Theta\right]
$$

and therefore $\mathbf{W}_{k}$ gets closer to the optimal weight vector $\mathbf{W}^{*}$.

The final form of the adaptive threshold is defined as

$$
\begin{aligned}
& f_{k}=\phi_{k}^{T} \mathbf{W}_{k} \\
& \mathbf{W}_{k}=\mathbf{W}_{k-1}-2 \mu\left(\gamma_{k}-e_{k}\right) \phi_{k}
\end{aligned}
$$

\subsection{ATLMS Tuning Parameters}

A fault flag is raised if the ATLMS crosses the insensitive residual $r_{0}(t)$. Therefore, the dynamics of the insensitive residual $r_{0}(t)$ must be taken into account in order to minimize false/missed alarms. For this reason, the parameter Safety Offset $\left(e_{0}\right)$ is set to prevent undesired false alarms during the transient response of $r_{0}(t)$ or to decrease the distance between the adaptive threshold and $r_{0}(t)$ in case of $r_{1}(t)$ signal is not very sensitive to the applied fault.

The Sensitivity Rate (a) describes the sensitivity of the adaptive threshold due to changes in the trend of the residuals as determined by the SPRT. In case of fault occurrence and for $a<0$, the adaptive threshold increases in the direction of the insensitive residual $r_{0}(t)$. Otherwise, the adaptive threshold increases in the opposite direction of the insensitive residual. This is a useful feature of ATLMS to avoid erroneous interpretations of adaptive threshold responses due to possible mistakes in the design of residual generators (when sensitive and insensitive residuals are incorrectly interchanged, for example), or by unexpected behavior of the residuals.

The ATLMS Convergence Rate $(\mu)$ is originally from the LMS algorithm and it is responsible for the adaptation stability and convergence speed of the ATLMS.

\section{Simulation Results and Discussion}

\subsection{Simulation Methodology}

The simulation methodology consists of three different flight scenarios with each of the proposed IMU faults presented in table 1 with following assumptions:

- Occurrence of single faults;

- Backup IMU does not fail;

- Reconfiguration procedure is defined as switching from primary IMU to standby/backup IMU once a fault is detected.

For the sake of simplification, all faults are introduced at 50 s. For incipient faults, the amplitude values described in table 1 refer to the slope of the faulty signals. For the extra noise faults, additive white noise is introduced with the indicated distribution. These faults cover both longitudinal and lateral aircraft dynamics and were chosen based on a priori knowledge and discussions with Elektra 2 Solar team. Due to the critical aspect of IMU faults, the fault detection flag is activated - and kept active - if any of the dedicated adaptive thresholds indicates a fault. Figure 4 illustrates the scheme of the bank of ATLMS for longidutinal dynamics. A similar 
scheme is used for the lateral dynamics. For the longitudinal measurements, the bank of ATLMS uses the yaw angle $\psi$ as the insensitive residual. For the lateral measurements, the bank of ATLMS uses the pitch angle $\theta$ as the insensitive residual.

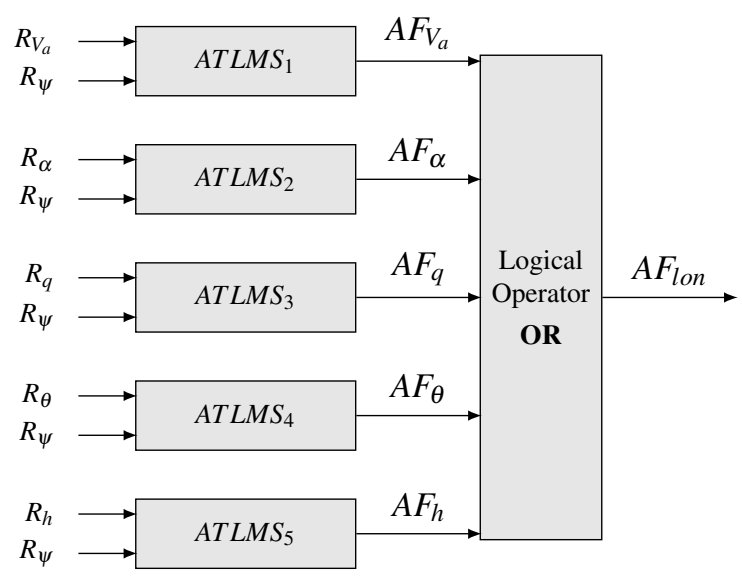

Figure 4: Bank of ATLMS for Longitudinal Dynamics. $R_{i}$ is the residual of measurement $i . V_{a}$ is the aircraft airspeed, $\alpha$ is the angle of attack, $q$ is the pitch rate, $\theta$ is the pitch angle, and $h$ is the aircraft altitude. The bank of ATLMS uses the yaw angle $\psi$ residual as the insensitive residual. The label $A F_{i}$ refers to the alarm flag of measurement $i$ and $A F_{l o n}$ is the combined alarm flag for longitudinal dynamics used for sensor switching reconfiguration in case of fault occurrence.

\subsection{Incipient fault in pitch angle $\theta$}

The first simulation scenario illustrates an incipient fault in pitch angle $\theta$ at $50 \mathrm{~s}$. As expected, a fault in pitch angle affects the longitudinal alarm flag Lon Flag but not the lateral alarm flag Lat Flag (fig 5 and fig. 6). The lateral adaptive thresholds present responses in the opposite direction of the insensitive residual. The overall aircraft performance would not remain in an acceptable flight condition if the reconfiguration action does not take place. As illustrated in figure 8, the aircraft airspeed/altitude suddenly increases/decreases in case of the proposed incipient fault occurs. This behavior could lead to overall system failure. However, due to the fault detection promptness and reconfiguration, the aircraft was slightly affected. Figure 7 presents the faulty and reconfigured aircraft roll, pitch and yaw angles.

\subsection{Extra noise fault in pitch rate $q$}

The second simulation scenario illustrates extra noise fault in angular rate $q$ at $50 \mathrm{~s}$. As expected, the proposed fault affects the longitudinal alarm flag Lon Flag but not the lateral alarm flag Lat Flag (fig 9 and fig. 10). Although the overall aircraft performance remains in an acceptable flight condition, the elevator control signal $\delta_{e}$ shows an undesired intermittent behavior (fig. 12). In the worst case scenario, this behavior could damage the elevator control surface and lead to overall system failure. However, due to the fault detection promptness and reconfiguration, the unpermitted behavior of the elevator surface is minimized. Figure 11 presents the faulty and

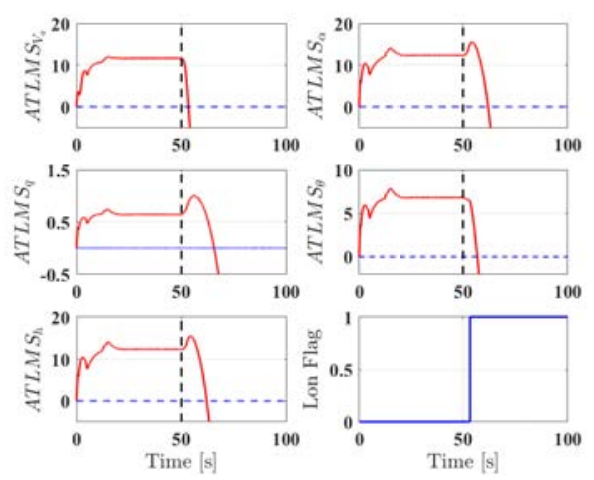

Figure 5: Dedicated adaptive thresholds and fault flag for longitudinal measurements with an incipient fault in pitch angle $\theta$.
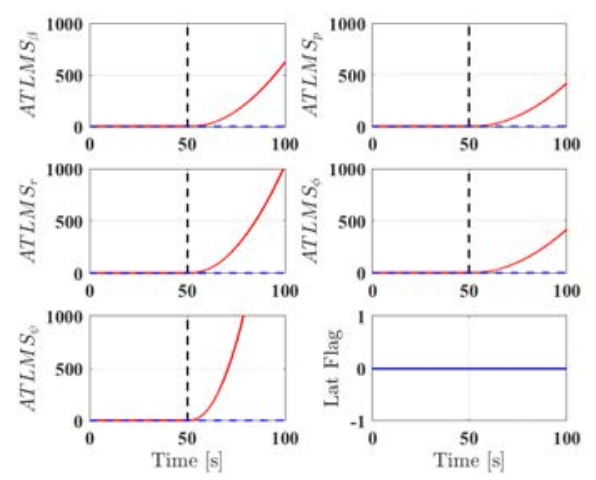

Figure 6: Dedicated adaptive thresholds and fault flag for lateral measurements with an incipient fault in pitch angle $\theta$.
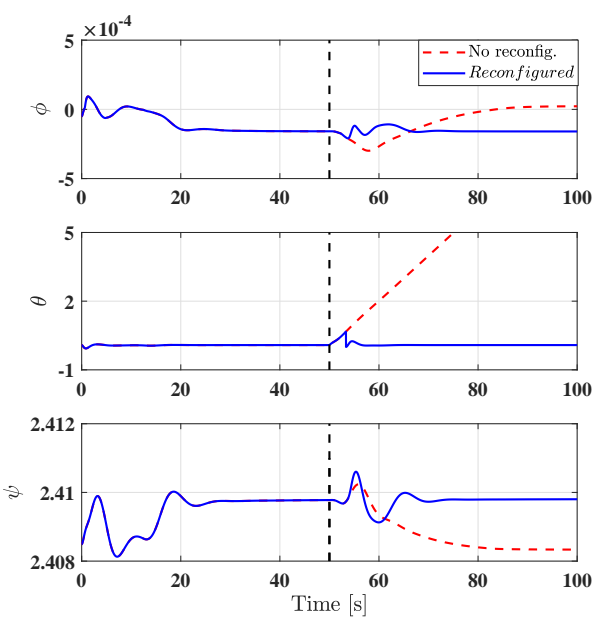

Figure 7: Faulty and reconfigured aircraft roll, pitch and yaw angles (rad) for an incipient fault in pitch angle $\theta$.

reconfigured aircraft angular rates $(\mathrm{rad} / \mathrm{s})$ for the second simulation scenario. 


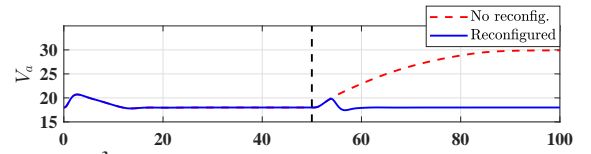

$1 \times 10^{-3}$
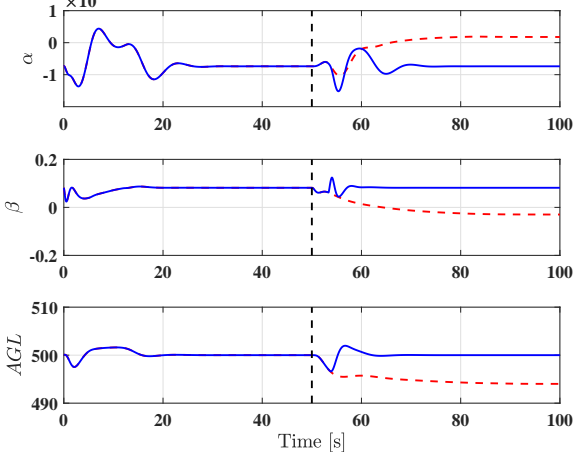

Figure 8: Faulty and reconfigured aircraft airspeed $(\mathrm{m} / \mathrm{s})$, aerodynamic angles $(\mathrm{rad})$, and altitude above ground $(\mathrm{m})$ for an incipient fault in pitch angle $\theta$.

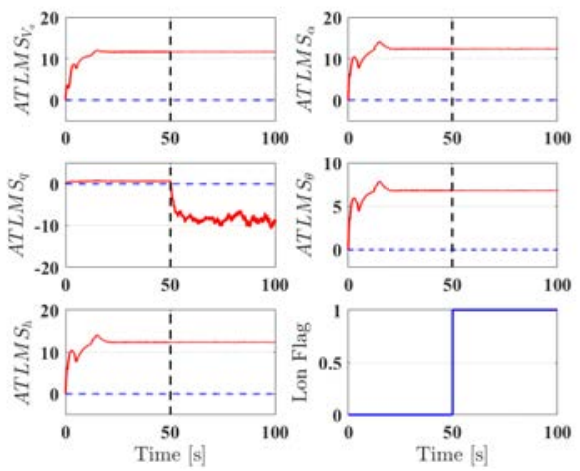

Figure 9: Dedicated adaptive thresholds and fault flag for longitudinal measurements with extra noise fault in angular rate q.

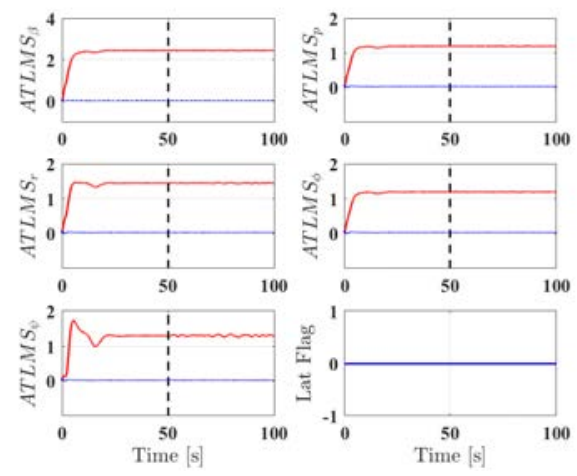

Figure 10: Dedicated adaptive thresholds and fault flag for lateral measurements with extra noise fault in angular rate $q$.
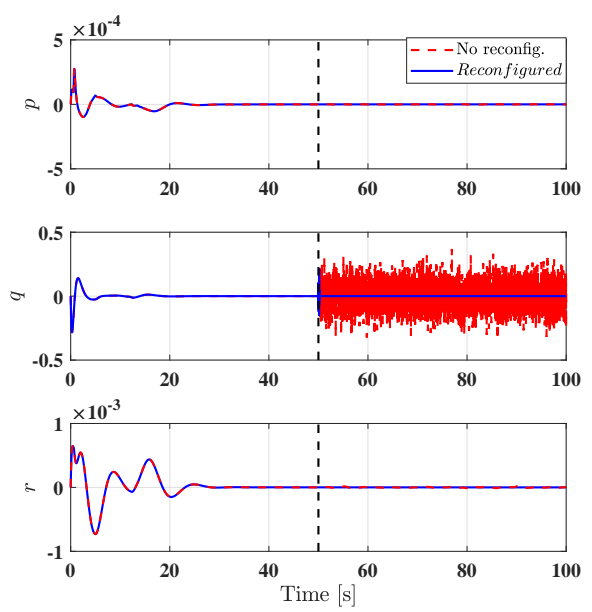

Figure 11: Faulty and reconfigured aircraft angular rates $(\mathrm{rad} / \mathrm{s})$ for extra noise fault in angular rate $q$.
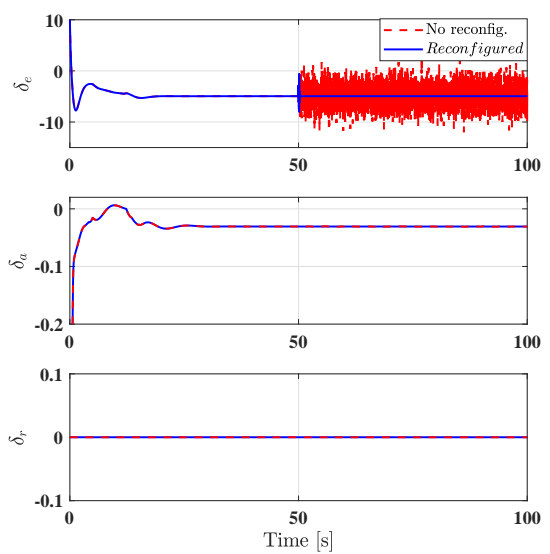

Figure 12: Faulty and reconfigured signals of aircraft control surfaces (degrees) for extra noise fault in angular rate $q$.

\subsection{Abrupt fault in roll angle $\phi$}

The third simulation scenario illustrates an abrupt fault in roll angle $\phi$ at 50s. As expected, a fault in roll angle affects the lateral alarm flag Lat Flag but not the longitudinal alarm flag Lon Flag (fig 14), since longitudinal and lateral linear models are decoupled as presented in equations (1) and (2). In the case of the proposed abrupt fault occurrence, the overall aircraft airspeed and altitude would remain in an acceptable flight condition even if the reconfiguration action does not take place (fig. 16. However, this is not the case for the aircraft attitude once roll and yaw angles would deviate from normal condition as illustrated in figure 15 . Figures 15 and 16 present the faulty and reconfigured aircraft attitude angles (rad), airspeed (m/s), aerodynamic angles ( $\mathrm{rad})$, and altitude above ground $(\mathrm{m})$. 


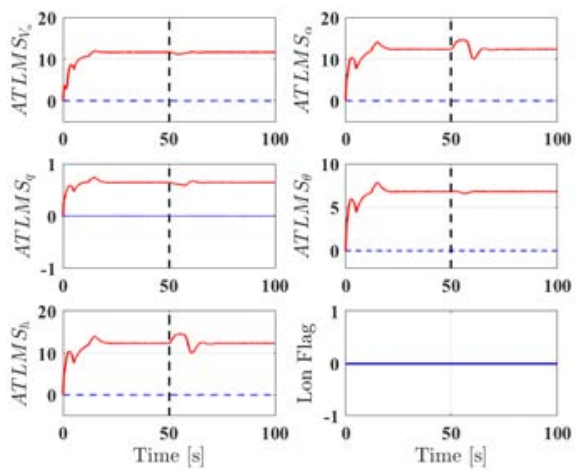

Figure 13: Dedicated adaptive thresholds and fault flag for longitudinal measurements with an abrupt fault in roll angle $\phi$.

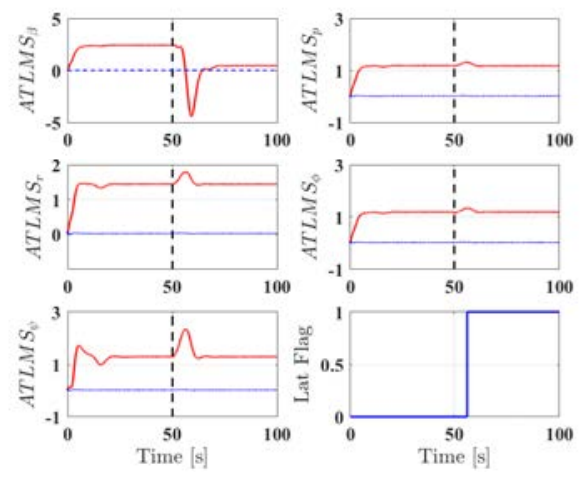

Figure 14: Dedicated adaptive thresholds and fault flag for lateral measurements with an abrupt fault in roll angle $\phi$.
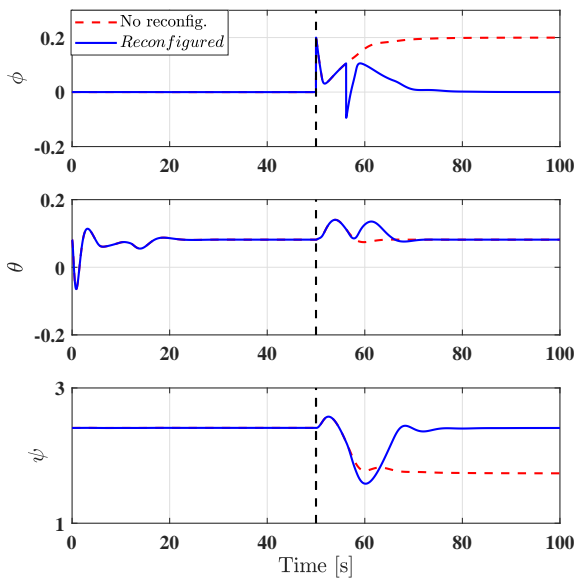

Figure 15: Faulty and reconfigured aircraft roll, pitch and yaw angles (rad) for an abrupt fault in roll angle $\phi$.

\section{Conclusion}

The main contribution of this work was the development of a model-based sensor fault detection and diagnosis approach to
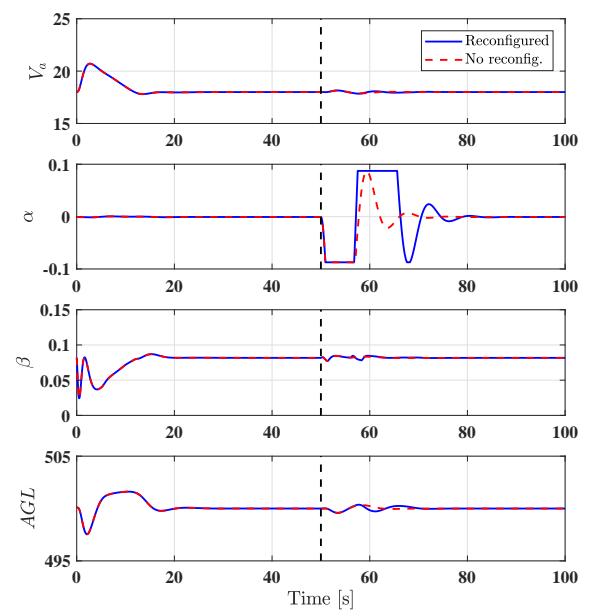

Figure 16: Faulty and reconfigured aircraft airspeed $(\mathrm{m} / \mathrm{s})$, aerodynamic angles ( $\mathrm{rad})$, and altitude above ground $(\mathrm{m})$ for an abrupt fault in roll angle $\phi$.

be used in the Elektra 2 Solar aircraft. An adaptive threshold (ATLMS) based on the Least Mean Squares algorithm was used to detect different types of faults in IMU measurements. One of the major advantages of the ATLMS technique is the possibility - not fully explored in this work - of parameter tuning. This may result in a better fault detection performance. Besides that, the adaptive threshold is able to to handle dynamic effects on the insensitive residual. Such effects may arise due to plant perturbations caused by control actions or due to the dynamic nature of the residual generator. As a consequence, non-zero residual values can be generated in faultfree scenario, resulting in a set of undesired false alarms. This drawback could also be minimized with suitable tuning of ATLMS parameters. Due to time constraints, the proposed FDD approach have not been integrated and tested in real flights yet.

\section{References}

[1] G. HEREDIA and A. OLLERO. Sensor fault detection in small autonomous helicopters using observer/kalman filter identification. In IEEE International Conference on Mechatronics, 2009.

[2] M. BASSEVILLE. Detecting changes in signals and systems - a survey. Annual Reviews in Control, 24:309-326, 1988.

[3] R. ISERMANN. Supervision, fault-detection and faultdiagnosis methods - an introduction. Control Engineering Practice, 5:639-652, 1997.

[4] R. ISERMANN. Model-based fault-detection and diagnosis - status and applications. Annual Reviews in Control, 29:71-85, 2005. 
[5] P. FRANK. Fault diagnosis in dynamic systems using analytical and knowledge-based redundancy. Automatica, 26:459-474, 1990 .

[6] R. PATTON and J CHEN. Observer-based fault detection and isolation: Robustness and applications. Control Engineering Practice, 5:671-682, 1997.

[7] A. CARVALHO. Fault detection, diagnosis and reconfiguration for optimized design of safe space vehicles applied to the PMM and planetary rovers. Ph.d. dissertation, Instituto Nacional de Pesquisas Espaciais (INPE), Sao Jose dos Campos, Brazil, 2012.

[8] R. ISERMANN. Fault-diagnosis Systems: An Introduction from Fault Detection to Fault Tolerance. Springer, 2006.

[9] S. SIMANI, C. FANTUZZI, and R. PATTON. Modelbased fault diagnosis in dynamic systems using identification techniques. Springer, 2003. 